\title{
Desordens temporomandibulares e dor orofacial: estudo da quali- dade de vida medida pelo Medical Outcomes Study 36 - Item Short Form Health Survey*
}

\author{
Temporomandibular disorders and orofacial pain: study of quality of life measured by the \\ Medical Outcomes Study 36 - Item Short Form Health Survey.
}

Denis Noboru Kuroiwa ${ }^{1}$, Juliano Gaspari Marinelli ${ }^{1}$, Marta Solange Rampani², Wagner de Oliveira², Denise Nicodemo ${ }^{3}$

* Recebido do Departamento de Odontologia Social e Clínica Infantil e Centro de Oclusão e Articulação Temporomandibular da Faculdade de Odontologia de São José dos Campos da Universidade Estadual Paulista Julio de Mesquita Filho (COAT/UNESP). São José dos Campos, SP.

- Bolsa FAPESP concedida Processo n 06/60923-1 - Iniciação Científica.

\section{RESUMO}

JUSTIFICATIVA E OBJETIVOS: O conhecimento de indicadores de qualidade de vida $(\mathrm{QV})$ relacionados à saúde bucal é especialmente relevante para a Odontologia considerando o impacto que as condições bucais podem provocar no bem estar psicológico e social. Estudos sobre aspectos psicossociais contribuem para maior integração da conduta clínica e assistencial, preocupação compartilhada com profissionais da saúde. Integrar as áreas de Psicologia e Odontologia quebrando paradigmas interdisciplinares e o interesse em conhecer os aspectos psicológicos dos pacientes, motivou a realização deste estudo.

1. Cirurgiões-Dentistas, Graduados pela Faculdade de Odontologia de São José dos Campos da Universidade Estadual Paulista Júlio de Mesquita Filho (UNESP). São José dos Campos, SP, Brasil.

2. Professores Doutores do Centro de Oclusão e Articulação Temporomandibular da Faculdade de Odontologia de São José dos Campos da Universidade Estadual Paulista Júlio de Mesquita Filho (UNESP). São José dos Campos, SP, Brasil.

3. Professora Doutora; Responsável pela Disciplina de Psicologia Aplicada à Odontologia, Faculdade de Odontologia de São José dos Campos da Universidade Estadual Paulista Júlio de Mesquita Filho (UNESP). São José dos Campos, SP, Brasil.

\section{Endereço para correspondência:}

Prof $^{a}$ Dra. Denise Nicodemo

Faculdade de Odontologia de São José dos Campos da UNESP

Departamento de Odontologia Social e Clínica Infantil.

Av. Engo Francisco José Longo, 777. Jardim São Dimas

12245-000 São José dos Campos, SP.

Fone: (12) 3947-9076

E-mail: denise@fosjc.unesp.br
O objetivo deste estudo foi avaliar a QV dos pacientes com disfunção temporomandibular e/ou dor orofacial.

MÉTODO: Foi aplicado o Questionário Genérico de Avaliação de Qualidade de Vida - Medical Outcomes Study 36 - Item Short Health Survey (SF-36) a 91 pacientes, que buscaram atendimento por apresentarem sinais e/ou sintomas de disfunção temporomandibular (DTM) e dor orofacial (DOF). O SF-36 avalia 8 domínios: capacidade funcional (CF), aspectos físicos (AF), dor, estado geral de saúde (EGS), saúde mental (SM), aspectos emocionais (AE), aspectos sociais (AS) e vitalidade (V).

RESULTADOS: A análise estatística descritiva e inferencial pela Correlação de Pearson ( $\mathrm{p}$-valor $\leq 0,05$ ) demonstrou, com exceção da capacidade funcional $(73,2)$, valores médios entre 50 e 64 para os demais domínios: AF - 57,6; Dor - 50; EGS - 54,5; V - 53,4; AS - 63,6; AE - 51,8; SM - 58. Considerando-se que a pontuação varia de 0 a 100 , ou seja, do pior para o melhor estado de saúde, os valores médios foram baixos. Verificou-se correlação entre CF e EGS (p-valor 0,01), tendência de significância para dor e EGS (p-valor 0,07).

CONCLUSÃO: Os aspectos dor e capacidade funcional interferem no estado geral de saúde; os pacientes com DTM e DOF sofreram impacto negativo na qualidade de vida pelo prejuízo dos aspectos físicos e mentais.

Descritores: Dor orofacial, Impacto psicossocial, Qualidade de vida.

\section{SUMMARY}

BACKGROUND AND OBJECTIVES: The understanding of quality of life (QL) indicators related to oral health 
is especially relevant for Dentistry, considering the impact that oral conditions may have in psychological and social well being. Studies on psychosocial aspects contribute for a better integration of clinical and assistance approach, which is a shared concern with health professionals. Integrating psychology and dentistry breaking interdisciplinary paradigms and the interest in knowing patients' psychological aspects, motivaded this study. This study aimed at evaluating the QL of patients with temporomandibular dysfunction and / or orofacial pain.

METHOD: The Generic Questionnaire to Evaluate Quality of Life - Medical Outcomes Study 36 - Item Short Health Survey (SF-36) was applied to 91 patients who looked for assistance due to signs and / or symptoms of temporomandibular dysfunction (DTM) and orofacial pain (OFP). SF-36 evaluates 8 domains: functional capacity (FC), physical aspects (FA), pain, general health status (GHS), mental health (MH), emotional aspects (EA), social aspects (AS) and vitality (V).

RESULTS: Descriptive and inferential statistical analysis by Pearson's Correlation $(\mathrm{p}=0.05)$ has shown, with the exception of functional capacity (73.2), mean values between 50 and 64 for remaining domains: PA - 57.6; Pain - 50; HGS - 54.5; V - 53.4; AS - 63.6; EA - 51.8; MH -58 . Considering that grades go from zero to 100 , that is, the worst and best health status, mean values were low. There has been correlation between FC and GHS ( $p=$ $0.01)$ and significance trend for Pain and GHS $(p=0.07)$.

CONCLUSION: Pain and functional capacity interfere with general health status; patients with TMD and OFP suffered negative impact in quality of life due to impairment of physical and mental aspects.

Keywords: Orofacial pain, Psychosocial impact, Quality of life.

\section{INTRODUÇÃO}

Podendo ser compreendida como um processo dualístico, a dor apresenta duas variantes: a percepção, que consiste em um processo anatomofisiológico, por meio do qual a dor é recebida e transmitida e reação, que se resume na manifestação da percepção do ser vivo diante do fenômeno.

A dor tanto facial quanto dentária é o aspecto mais citado dentre os indicadores da saúde bucal que impactam a qualidade de vida (QV), seguido do prejuízo das horas de sono e dos problemas mastigatórios ${ }^{1}$. A dor orofacial pode alterar a QV mais do que outras condições sistêmicas, tais como diabetes, hipertensão ou úlcera. Indivíduos nessa condição vivenciam grandes mudanças no seu dia a dia, incluindo: perda de dia de trabalho, ausência de rela- cionamento com familiares e amigos, insatisfação com a condição bucal, ingestão de medicamento e modificação na dieta alimentar ${ }^{2}$.

É aceita pela Academia Americana de Dor Orofacial que Desordem Temporomandibular é um termo coletivo envolvendo um número de sinais e sintomas que incluem ruídos articulares, como estalidos e crepitação; dor nos músculos mastigatórios; limitação dos movimentos mandibulares; dores faciais; dores de cabeça e dores na articulação temporomandibular (ATM) ${ }^{3,4}$. O termo é sinônimo de disfunções temporomandibulares (DTM) as quais têm sido identificadas como a maior causa de dor não dental na região orofacial.

Por ser ainda bastante controverso, muito se tem estudado sobre a etiologia da DTM e da DOF, visto que frequentemente diagnóstico e tratamento são de caráter multidisciplinar $^{5}$. Vários são os fatores considerados na literatura como causadores ou potencializadores de DTM e DOF: desarmonias oclusais, perfil psicológico, lesões musculoesqueletais, hábitos parafuncionais, estresse emocional, lassidão do tecido conjuntivo, trauma, fatores anatômicos e patofisiológicos ${ }^{6-11}$.

As DTM encontram no bruxismo um forte incremento para a sua instalação, uma vez que este hábito atua intensamente na predisposição e na suscetibilidade dos pacientes no que se refere à etiologia e agravamento dos quadros de DTM ${ }^{12}$. Este tipo de inter-relação entre bruxismo e DTM pode ser ilustrado por inúmeros estu$\operatorname{dos}^{13-17}$. Estes estudos afirmaram haver uma relação direta entre a incidência e a prevalência do bruxismo e das DTM, ainda que o bruxismo seja um forte agravante na DTM, podendo aumentar em até $40 \%$ a sintomatologia dolorosa dos quadros.

As primeiras associações entre as DTM e fatores psicológicos surgiram a partir de estudos de uma psiquiatra e um cirurgião-dentista, trabalhando conjuntamente ${ }^{18}$. Dentre os fatores psicológicos associados à etiologia das DTM, foram relacionados os comportamentais, os cognitivos e os emocionais ou afetivos, como a ansiedade, o estresse e a depressão.

O grupo de Qualidade de Vida da Organização Mundial de Saúde (OMS) definiu qualidade de vida como a percepção de um indivíduo de sua posição na vida no contexto da cultura e do sistema de valores em que vive e em relação aos seus objetivos, expectativas, padrões e preocupações ${ }^{19}$. Os indicadores de QV podem ser especialmente relevantes para a Odontologia considerando a importância da aparência facial na autoimagem e autoestima e também o impacto, por vezes sutil, que as condições bucais podem provocar no bem estar e funcionamento psicológico ${ }^{20-22}$. 
Espera-se, com este estudo, contribuir com a conduta clínica e assistência cada vez mais integradas e direcionadas a promoção do "bem estar físico, psíquico e social" pela aplicabilidade do conhecimento científico na prática profissional. Portanto, objetivou-se estudar a QV dos pacientes que buscam por uma melhora da saúde bucal por apresentarem DTM e/ou dor orofacial.

O objetivo deste estudo foi avaliar QV dos pacientes com DTM e/ou DOF.

\section{MÉTODO}

Após aprovação pelo Comitê de Ética da Universidade Estadual Paulista Júlio de Mesquita Filho, São José dos Campos sob protocolo no 06/2005-PH/CEP, realizou-se este estudo com 91 pacientes adultos de ambos os sexos, inscritos para atendimento no Centro de Oclusão e Articulação Temporomandibular (COAT) da Faculdade de Odontologia de São José dos Campos por apresentarem sinais e/ou sintomas de DTM e DOF, sendo estes os critérios de inclusão. $\mathrm{Na}$ inscrição para tratamento no COAT, os pacientes são examinados por um único examinador, a fim de confirmar os sinais e sintomas relacionados à dor orofacial de DTM. Essas pessoas aguardam em uma lista para diagnóstico de DTM e tratamento específico e a partir desta lista é que foram recrutados para este estudo. Quanto aos critérios de inclusão, os pacientes deveriam ter, pelo menos, um dos seguintes sinais ou sintomas por um período mínimo de três meses: a dor dos músculos mastigatórios, dor na ATM, através da escala analógica visual $(\mathrm{EAV})>5$, ruído articular e limitação funcional. Pacientes com doenças sistêmicas que pudessem afetar a área orofacial ou incapacidade cognitiva para responder ao questionário SF-36 foram excluídos da amostra.

Os pacientes participaram da pesquisa voluntariamente mediante consentimento livre e esclarecido, assinado por cada participante, no momento em que se apresentaram no COAT, após serem inscritos e convocados pelo Setor de Triagem da Unidade.

\section{Procedimento}

O procedimento constou da aplicação do Questionário Genérico de Avaliação de Qualidade de Vida - Medical Outcomes Study 36-Item Short Form Health Survey (SF$36)^{23}$. A aplicação foi realizada pelo pesquisador responsável pelo estudo de forma coletiva e assistida, em local apropriado.

\section{Instrumento}

O SF-36, validado para a cultura brasileira ${ }^{24}$ em 1999, consiste de 11 perguntas fechadas e tem como propósito avaliar 8 domínios divididos em 2 grupos: o físico, envolvendo a capacidade funcional, aspectos físicos, dor e estado geral da saúde; e o Mental envolvendo saúde mental, aspectos emocionais, sociais e vitalidade. A pontuação varia de 0 a 100 , ou seja, do pior para o melhor estado e saúde.

O questionário enfatiza a percepção do indivíduo sobre sua saúde nas últimas quatro semanas. Apresenta-se, a seguir, a descrição do que pretende avaliar cada um dos domínios:

- Capacidade funcional: avalia como o indivíduo realizou suas tarefas diárias habituais (10 itens presentes na questão 3);

- Aspectos físicos: avalia como a saúde física interferiu nas atividades domésticas ou profissionais habituais (quatro itens presentes na questão 4);

- Dor: avalia quanta dor o indivíduo sentiu nas últimas quatro semanas e as limitações que ela provocou em seu cotidiano (dois itens presentes nas questões 7 e 8);

- Estado geral de saúde: avalia a percepção que o indivíduo tem de sua própria saúde e sua expectativa em relação ao futuro (cinco itens presentes nas questões 1 e 11);

- Vitalidade: avalia o grau de energia e disposição do indivíduo para realizar suas tarefas diárias (quatro itens presentes na questão 9);

- Aspectos sociais: avalia o quanto as atividades sociais habituais do indivíduo foram afetadas por seu estado físico ou emocional (dois itens presentes nas questões 6 e 10);

- Aspecto emocional: avalia como o estado emocional interferiu nas atividades diárias domésticas ou no trabalho (três itens presentes na questão 5);

- Saúde mental: avalia quanto tempo o indivíduo tem se sentido ansioso e deprimido ou feliz e tranquilo no seu cotidiano (cinco itens presentes na questão 9).

Foram realizadas as análises estatísticas descritiva e inferenciais pelo cálculo dos coeficientes de Correlação Linear de Pearson, adotando-se nível de significância de 5\%.

\section{RESULTADOS}

Verificou-se que dentre os 91 pacientes que buscaram atendimento no COAT, 73 eram do sexo feminino, $80,22 \%$ e 18 do sexo masculino, 19,78\% (Gráfico 1).

Os resultados do SF-36 demonstraram, com exceção da capacidade funcional que apresentou média de 73,57, 


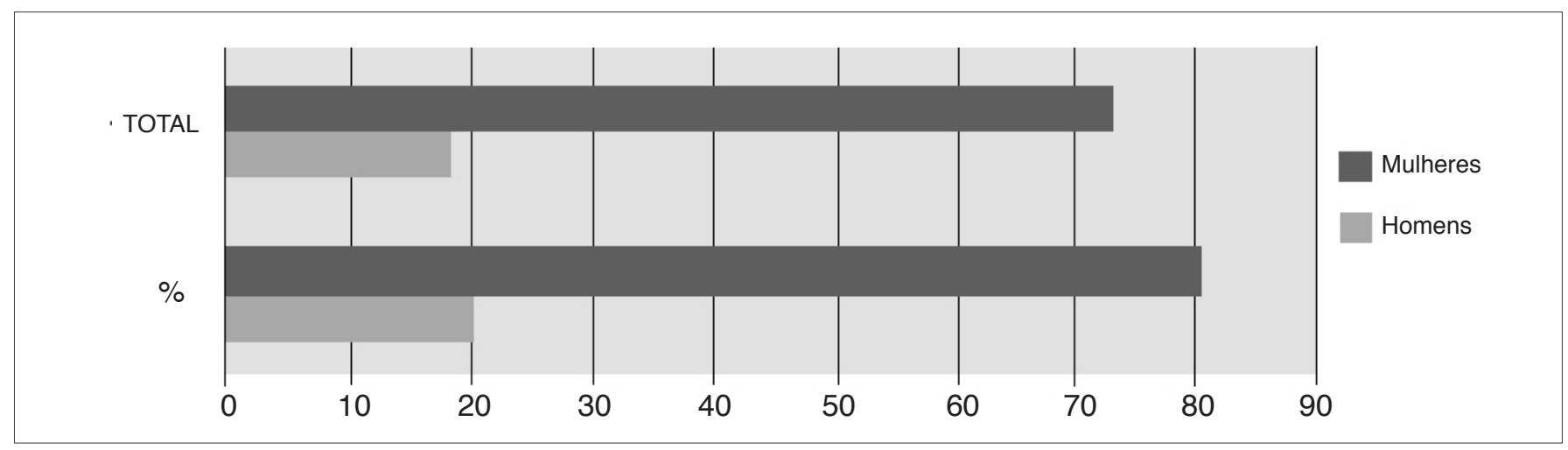

Gráfico 1 - Número de atendimentos por sexo

valores médios entre 52 e aproximadamente 64 para os demais domínios, ou seja, valores baixos, indicando um comprometimento na QV destes pacientes. Os domínios capacidade funcional e aspectos sociais $(73,57$ e 63,55, respectivamente) que avaliam realização das tarefas diárias habituais como subir degraus, abaixar-se, levantar-se e andar pequenas distâncias, assim como as atividades sociais não foram significativamente afetados. Entretanto, os domínios dor, aspecto emocional e vitalidade que apresentaram, respectivamente, os menores valores, 52,12; 52,56 e 53,41 comprovam que atividades diárias normais no lar e no trabalho foram afetadas pelo comprometimento emocional e físico, demonstradas por maior indisposição, cansaço e fadiga. Verificaram-se também, por meio do domínio aspectos físicos $(58,32)$, comprometimento de trabalhos e tarefas diárias (Tabela 1, Gráfico 2).

\section{DISCUSSÃO}

Os resultados obtidos neste estudo demonstraram significativa predominância de pacientes do sexo feminino (80,22\% versus $19,78 \%$ de pacientes do sexo masculino). Esta maior procura já havia sido sugerida em estudo que evidenciou que as mulheres tendem a apresentar a percepção da dor mais exata do que os homens ${ }^{25}$.

À semelhança, outros estudos propuseram que as DTM fazem parte de um espectro de síndromes associadas ao estresse e são caracterizadas por alterações somáticas e psicológicas, incluindo fadiga, prejuízo das atividades do trabalho e escola, distúrbios do sono e do apetite/alimentação, ansiedade e depressão ${ }^{26,27}$. Níveis mais altos de dor e estresse foram encontrados em pacientes com DTM em comparação ao grupo controle ${ }^{26}$.

Tabela 1 - Análise descritiva de cada domínio avaliado pelo SF-35

\begin{tabular}{|l|c|c|c|c|c|c|c|c|}
\hline SF-36 & CF & AF & Dor & EGS & Vitalidade & AS & AE & SM \\
\hline$>$ Valor & 100 & 100 & 94 & 97 & 95 & 100 & 100 & 96 \\
\hline$<$ Valor & 10 & 0 & 0 & 10 & 0 & 0 & 0 & 0 \\
\hline Média & 73,57 & $58,32^{*}$ & 52,12 & $54,78^{*}$ & 53,41 & 63,55 & 52,56 & 57,64 \\
\hline
\end{tabular}

* correlação entre os domínios (p-valor 0,01 )

$\mathrm{CF}=$ capacidade funcional; $\mathrm{AF}=$ aspecto físico; $\mathrm{EGS}=$ estado geral de saúde; $\mathrm{AS}=$ aspectos sociais; $\mathrm{AE}=$ aspectos emocionais; $\mathrm{SM}=$ saúde mental

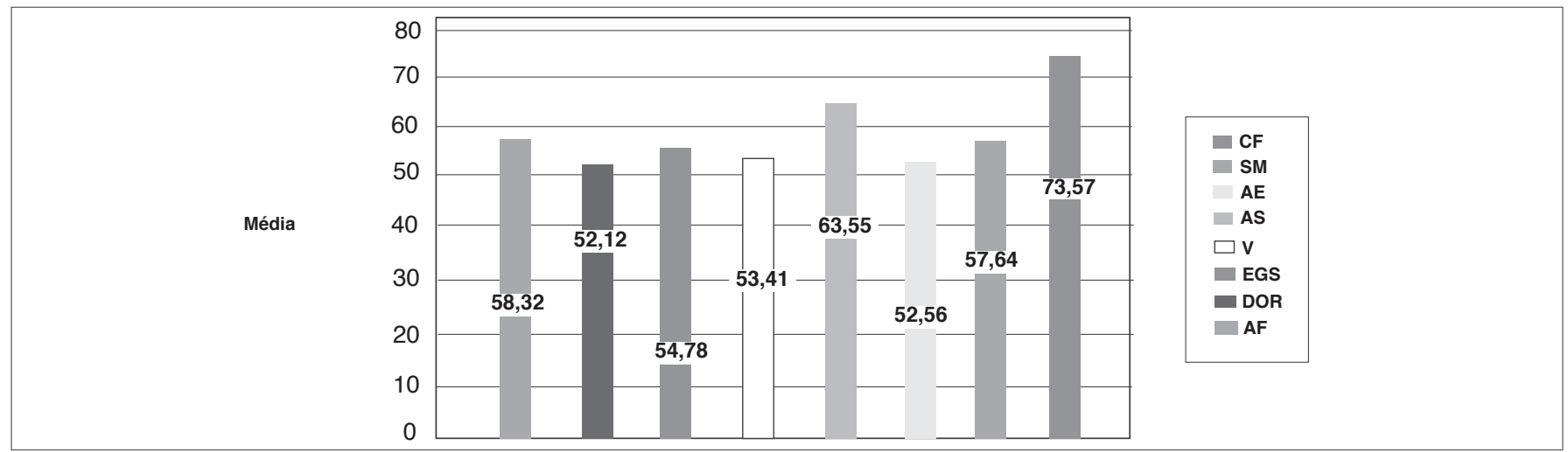

Gráfico 2 - Pontuação média de cada domínio do SF-36

$\mathrm{CF}=$ capacidade funcional; $\mathrm{SM}=$ saúde mental; $\mathrm{AE}=$ aspectos emocionais; $\mathrm{AS}=$ aspectos sociais; $\mathrm{EGS}=$ estado geral de saúde; $\mathrm{AF}=$ aspecto físico 
O domínio saúde mental, com média geral baixa $(57,63)$, demonstrou baixo controle emocional dos pacientes, representado, pelo que avalia este domínio, maior ansiedade, nervosismo e depressão. Os pacientes acreditam que sua saúde está ruim e tende a piorar; fato este, comprovado pela média do domínio estado geral de saúde $(54,78)$. Verificou-se ainda, correlação entre os domínios Aspectos Físicos e Estado Geral de Saúde (p-valor 0.01) e tendência de significância estatística para Dor e Estado Geral de Saúde (p-valor 0.07). Estudo ${ }^{28}$ demonstrou que a melhora de aspectos da saúde bucal podem interferir, positivamente, na condição física percebida por melhor desempenho das atividades de trabalho e do dia a dia.

Essa interferência negativa do bem estar físico, funcional, social, mental e psicológico, verificada a partir da aplicação do questionário de qualidade de vida SF-36, confirma o pressuposto previamente descrito, o qual ressalta que saúde não é meramente a ausência de doença ou enfermidade ${ }^{1}$.

Evidenciando-se mais uma vez, e neste estudo por intermédio do SF-36, que as DTM e DOF são desordens que apresentam uma etiologia multifatorial, deve-se atentar para o tratamento multidisciplinar, composto por vários profissionais como: cirurgiões dentistas, psicólogos, fonoaudiólogos, fisioterapeutas, neurologistas, para promover o melhor tratamento a este paciente e consequentemente melhorar a sua QV. Entretanto, estes indicadores subjetivos não devem ser usados no diagnóstico de doenças ou em substituição ao exame clínico, pois não fornecem os sinais objetivos da doença. Estes dados devem ser usados mais como um instrumento de avaliação, complementando as informações clínicas e possibilitando identificar pessoas que necessitem de ações curativas, preventivas ou educativas.

\section{CONCLUSÃO}

Os aspectos dor e capacidade funcional interferiram negativamente no estado geral de saúde dos pacientes com DTM e DOF; os pacientes com DTM e DOF sofreram impacto negativo na qualidade de vida pelo prejuízo dos aspectos físicos e mentais.

\section{REFERÊNCIAS}

1. Locker D, Grushka M. The impact of dental and facial pain. J Dent Res 1987;66(9):1414-7.

2. Biazevic MGH, Araújo ME, Michel-Crosato E. Indicadores de qualidade de vida relacionados com a saúde bucal: revisão sistemática. Rev Odontol
2002;4(1):13-25.

3. Parker MW. A dynamic model of etiology in temporomandibular disorders. J Am Dent Assoc 1990;120(3):283-90.

4. Bocchi EA, Kuhn AMB, Nascimento RSGF. Características psicológicas de pacientes com queixa de disfunção da articulação temporomandibular. Psikhe 2000;5(1):70-6.

5. McNeill C. Management of temporomandibular disorders: concepts and controversies. J Prosthet Dent 1997;77(5):510-22.

6. Riolo ML, Brandt D, TenHave TR. Associations between occlusal characteristics and signs and symptoms of TMJ dysfunction in children and young adults. Am J Orthod Dentofacial Orthop 1987;92(6):467-77. 7. Pullinger AG, Seligman DA. Trauma history in diagnostic groups of temporomandibular disorders. Oral Surg Oral Med Oral Pathol 1991;71(5):529-34.

8. Henrikson T, Ekberg EC, Nilner M. Symptoms and signs of temporomandibular disorders in girls with normal occlusion and Class II malocclusion. Acta Odontol Scand 1997;55(4):229-35.

9. Ruf S, Cecere F, Kupfer J, et al. Stress-induced changes in the functional electromyographic activity of the masticatory muscles. Acta Odontol Scand 1997;55(1):44-8.

10. Steed PA. Etiological factors and temporomandibular treatment outcomes: the effects of trauma and psychological dysfunction. Funct Orthod 1997; 14(4)17-22.

11. Rauhala K, Oikarinen KS, Raustia AM. Role of temporomandibular disorders (TMD) in facial pain: occlusion, muscle and TMJ pain. Cranio 1999; 17(4):254-61.

12. Santos A, Bergantin A, Maekawa M, et al. Análise crítica da participação dos fatores odontológicos e psicológicos na etiologia do bruxismo. Rev Odontológica de Araçatuba 2007;28(2):20-4.

13. Tsolka P, Walter JD, Wilson RF, et al. Occlusal variables, bruxism and temporomandibular disorders: a clinical and kinesiographic assessment. J Oral Rehabil 1995;22(12):849-56.

14. Lobbezoo F, Lavigne GJ. Do bruxism and temporomandibular disorders have a cause- and-effect relationship? J Orofac Pain 1997;11(1):15-23.

15. Molina OF, dos Santos Junior J, Nelson SJ, et al. A clinical study of specific signs and symptoms of CMD in bruxers classified by the degree of severity. Cranio 1999; 17(4):268-79.

16. Molina OF, Santos J, Mazzeto M, et al. Oral jaw 
behaviors in TMD and bruxism: a comparison study by severity of bruxism. Cranio 2001;19(2):114-22.

17. Venâncio RA, Camparis CM. Estudo da relação entre fatores psicossociais e desordens temporo-mandibulares. Rev Bras Odontol 2002;59(3):152-4.

18. Moulton RE. Psychiatric considerations in maxillofacial pain. J Am Dent Assoc 1955;51(4):408-14. 19. Power M, Harper A, Bullinger M. The World Health organization WHOQOL-100: tests of the universality Quality of Life in 15 different cultural groups worldwide. Health Psychol 1999;18(5):495-505.

20. Testa MA, Simonson DC. Assessment of quality-of-life outcomes. N Engl J Med 1996;334(13):835-40. 21. Nicodemo D, Pereira MD, Ferreira LM. Self-esteem and depression in patients presenting angle class III malocclusion submitted for orthognathic surgery. Med Oral Patol Oral Cir Bucal 2008;13(1):E48-51.

22. Barros Vde M, Seraidarian PI, Côrtres MI, et al. The impact of orofacial pain on the quality of life of patients with temporomandibular disorder. J Orofac Pain 2009;23(1):28-37.

23. Ware JE Jr, Sherbourne CD. The MOS 36item short-form health survey (SF-36). Med Care
1992;30(6):473-83.

24. Ciconelli RM, Ferraz MB, Santos W, et al. Tradução para a língua portuguesa e validação do questionário genérico de qualidade de vida SF-36 (Brasil SF-36). Rev Bras Reumatol 1999;39(3):143-50.

25. Giddon DB, Mosier M, Colton T, et al. Quantitative relationship between perceived and objective need for health care--dentistry as a model. Public Health Rep 1976;91(6):508-13.

26. Moody PM, Calhoun TC, Okeson JP, et al. Stress-pain relationship in MPD syndrome patients and non-MPD syndrome patients. J Prosthet Dent 1981;45(1):84-8.

27. Oliveira AS, Bermudez CC, Souza RA, et al. Impacto da dor na vida de portadores de disfunção temporomandibular. J Appl Oral Sci 2003;11(2):138-43.

28. Nicodemo D, Pereira MD, Ferreira LM. Effect of orthognathic surgery for class III correction on quality of life as measured by SF-36. Int J Oral Maxillofac Surg 2008;37(2):131-4.

Apresentado em 14 de fevereiro de 2011.

Aceito para publicação em 06 de junho de 2011. 